\title{
Pharmacist-based Donepezil Outpatient Consultation Service to improve medication persistence
}

This article was published in the following Dove Press journal:

Patient Preference and Adherence

27 August 2012

Number of times this article has been viewed

\author{
Norio Watanabe $e^{1,2}$ \\ Keiko Yamamura ${ }^{3}$ \\ Yusuke Suzuki ${ }^{4}$ \\ Hiroyuki Umegaki ${ }^{4}$ \\ Katsuro Shigeno ${ }^{5}$ \\ Ryo Matsushita' \\ Yoshimichi Sai' \\ Ken-ichi Miyamoto' \\ Kiyofumi Yamada ${ }^{6}$ \\ 'Department of Clinical Pharmacy, \\ Graduate School of Natural \\ Science and Technology, Kanazawa \\ University, Kanazawa, Ishikawa, Japan \\ ${ }^{2}$ Department of Pharmacy, Hashima \\ Municipal Hospital, Hashima, Gifu, \\ Japan; ${ }^{3}$ Department of Clinical \\ Pharmacy, School of Pharmacy, Aichi \\ Gakuin University, Nagoya, Aichi, \\ Japan; ${ }^{4}$ Department of Community \\ Healthcare and Geriatrics, Nagoya \\ University Graduate School of \\ Medicine, Nagoya, Aichi, Japan; \\ ${ }^{5}$ Department of Pharmacy Practice \\ and Sciences, School of Pharmacy, \\ Aichi Gakuin University, Nagoya, \\ Aichi, Japan; ${ }^{6}$ Department of \\ Neuropsychopharmacology and \\ Hospital Pharmacy, Nagoya University \\ Graduate School of Medicine, Nagoya, \\ Aichi, Japan
}

Correspondence: Keiko Yamamura I-100 Kusumoto-cho, Chikusa-ku, Nagoya, Aichi 464-8650, Japan

Tel +8I 527576767

Fax +8I 527576799

Email keiko-y@dpc.agu.ac.jp
Aim: Donepezil is widely used to delay the progression of cognitive dysfunction in patients with Alzheimer's disease (AD), but the efficacy of pharmacotherapy is often reduced by poor adherence to medication. In order to improve adherence by providing information about AD and the significance of pharmacotherapy, the Donepezil Outpatient Consultation Service (DOCS) was set up. The influence of this service on medication persistence was assessed in the present study.

Methods: Among outpatients starting donepezil therapy, we enrolled 59 patients between April 2008 and September 2010 before establishment of the DOCS (non-DOCS group) and 52 patients between October 2010 and March 2012 who attended the DOCS (DOCS group). Each patient's and their caregiver's understanding about the clinical features of AD and pharmacotherapy with donepezil were also assessed. Their understanding was compared before and after the DOCS, and the 1-year medication persistence rate and the reasons for discontinuation were also investigated.

Results: The 1-year medication persistence rate was significantly higher in the DOCS group than in the non-DOCS group $(73.1 \%$ vs $49.2 \%, P=0.008)$. We examined the association of medication persistence with age, sex, clinical dementia rating, living alone, and attending the DOCS. As a result, medication persistence was significantly higher in patients attending the DOCS. The main reasons for discontinuation of donepezil were transfer elsewhere (11) and gastrointestinal side effects (5) in the non-DOCS group, and transfer (9) and gastrointestinal side effects (3) in the DOCS group. The overall score for understanding was $2.5 \pm 1.7$ before attending the DOCS and it increased significantly to $5.7 \pm 0.7$ afterward $(P<0.001)$.

Conclusion: The DOCS consultation provided by hospital pharmacists for AD patients and their caregivers improved understanding about the clinical features of dementia and provided pharmacological knowledge about antidementia drugs, leading to better adherence to pharmacotherapy that could maximize its effect.

Keywords: Alzheimer's disease, cholinesterase inhibitors, consultation, donepezil, patient knowledge, pharmacists

\section{Introduction}

Alzheimer's disease (AD) is a progressive neurodegenerative illness with cognitive dysfunction as its core symptom. The number of patients with AD is increasing in Japan along with aging of the population. Achieving cure or complete remission of neurodegenerative diseases is difficult and only symptomatic therapy is available for AD.

Patients with AD show reduced brain levels of the neurotransmitter acetylcholine. Acetylcholine esterase is an enzyme that breaks down acetylcholine, and selective 
inhibitors of this enzyme are thought to activate cholinergic nerves by preventing the decomposition of acetylcholine and increasing its concentration in the synaptic cleft.

Accordingly, cholinesterase inhibitors are commonly used to treat the core symptoms of AD. Donepezil hydrochloride (donepezil) was the first of these drugs to be approved in Japan and has long been used as the main medication for AD. Donepezil has been shown to improve cognitive function for a certain period after the start of administration and also delays the progression of $\mathrm{AD}^{1,2}$ In addition to improving cognitive function, a number of reports have indicated that donepezil can improve the daily activities of $\mathrm{AD}$ patients ${ }^{3}$ and alleviate the burden on caregivers (eg, by reducing the need for supervision). ${ }^{4}$

We previously carried out a survey on persistence with donepezil therapy for AD patients at the Geriatric Outpatient Department of Nagoya University Hospital, and we found that only $50 \%$ of patients remained on treatment after 1 year. Patients generally discontinued treatment because the drug was considered to be ineffective or because of gastrointestinal symptoms. The medication persistence rate decreased as the severity of AD (assessed by the clinical dementia rating [CDR]) increased, suggesting that the therapeutic effect of this drug was not being fully exploited. ${ }^{5}$ The low persistence rate may also have occurred because family members/ caregivers had unrealistic expectations about this drug (such as hoping for improvement of cognitive function or reduction of the caregiver's burden) that exceeded its actual effects. Moreover, patients and their caregivers may have been excessively concerned about side effects such as gastrointestinal symptoms, leading to reduced adherence. ${ }^{5}$

Under such circumstances, the Department of Geriatrics and Hospital Pharmacy Department of Nagoya University Hospital, in cooperation with the School of Pharmacy at Aichi Gakuin University, has provided the Donepezil Outpatient Consultation Service (DOCS). The DOCS aims to increase understanding of dementia and its treatment with donepezil by $\mathrm{AD}$ patients and their caregivers in order to improve adherence, and is mainly run by pharmacists who are specially trained for this purpose.

The present study was performed to examine the effectiveness of the DOCS. Patients and their caregivers were surveyed to assess changes of their understanding about $\mathrm{AD}$ and donepezil treatment. In addition, the medication persistence rate was monitored during follow up and patient background factors with a possible influence on persistence were investigated, as were the reasons for discontinuing donepezil therapy.

\section{Methods}

\section{Subjects}

All of the subjects of this study had AD according to the criteria in the Diagnostic and Statistical Manual of Mental Disorders of the American Psychiatric Association. ${ }^{6}$

The DOCS is an advisory service provided by pharmacists at our hospital for AD patients and their family members/ primary caregivers. It was established in October 2010. A total of 59 patients ( 15 men and 44 women with a mean age of 79.0 years), who started therapy with donepezil during the period from April 2008 to September 2010 before the DOCS was established, were enrolled as the non-DOCS group. The CDR score was 0.5 in 2 patients, 1 in 42 patients, 2 in 10 patients, and 3 in 5 patients. Four patients lived alone and 55 lived with family members.

In addition, AD patients who attended the DOCS after it was established and received advice from a pharmacist were enrolled as the DOCS group. This group comprised 52 patients (21 men and 31 women with an average age of 77.2 years) attending the DOCS between October 2010 and March 2012. The CDR score was 0.5 in 14 patients, 1 in 23 patients, 2 in 9 patients, and 3 in 6 patients. Seven patients lived alone and 45 lived with family members. Persistence with donepezil therapy was investigated in both the DOCS group and the non-DOCS group.

In addition, changes in understanding the clinical features of $\mathrm{AD}$ and pharmacotherapy with donepezil were investigated in patients and their family members who attended the DOCS. Patient characteristics that had an influence on continuation of treatment were also assessed. In all patients from both groups, donepezil was started at a dose of $3 \mathrm{mg} /$ day and was increased to $5 \mathrm{mg} /$ day after 1 or 2 weeks.

\section{Donepezil Outpatient Consultation Service}

The DOCS provides advice to outpatients of the Department of Geriatrics of Nagoya University Hospital who are prescribed donepezil and their family members. If patients or family members wished to attend the DOCS after it was recommended by their primary physician at the outpatient clinic, an application was completed at the Hospital Pharmacy Department and an appointment was made. A pharmacist provided advice to each patient/family in the drug consultation room to protect their privacy.

Before the consultation, information that included the date of starting donepezil therapy, the dose, and the living status (ie, living independently or with the family) was obtained to gain a better understanding of each patient's situation. In addition, the severity of cognitive dysfunction 
was assessed from the CDR based on data in the electronic medical record.

Background factors that might influence medication persistence were also ascertained.

Information related to use of donepezil was collected, including adherence to donepezil therapy, the timing of drug intake, and the patient's swallowing function. We asked the patient and caregiver about swallowing of medications and food because an association between dementia and dysphagia has been reported. ${ }^{7}$ We also provided the following instructions about dosing. (1) The primary caregiver should supervise the patient taking donepezil and store the drug. (2) The time of administration should be set according to when the patient can easily receive the drug or when it is convenient for the family member because meals have no influence on absorption. (3) Donepezil should be dissolved in water, barley tea, or orange juice if the patient does not want to take it because of not understanding that he/she has dementia or denial of the disease. Then the patients and their caregivers were evaluated to assess their understanding of the clinical features of AD and knowledge about donepezil.

If any misunderstanding or lack of knowledge about the patient's condition was revealed by the survey, sufficient time was taken to provide the caregiver with information using a textbook devised for the DOCS.

The following points were explained to the patients and their family members. (1) Donepezil shows efficacy from about three months after the start of treatment. (2) It can improve cognitive function for a certain period and delays subsequent progression of the disease. (3) It can also prevent loss of activities of daily living and reduce the need to supervise patients. (4) Importantly, if there is no change of symptoms, this is because the drug is delaying disease progression. ${ }^{1-4}$ (5) Better adherence not only improves the quality of life of patients, but also reduces the burden on caregivers.

Four weeks after the first DOCS consultation, the survey was repeated to assess how well the information provided at the first session had been understood. If some points were not clear, further instruction was given based on the textbook.

\section{Survey of understanding}

In consultation with specialist geriatricians, we prepared a 6-item survey for caregivers to assess their understanding about the clinical features of AD and pharmacotherapy with donepezil (Table 1).

Questions 1 and 2 covered the difference between forgetfulness and dementia and whether dementia is a pathologic condition. Question 3 was about the effects of donepezil,
Table I Survey of understanding about the clinical features of Alzheimer's disease and donepezil therapy for patients and primary caregivers

\begin{tabular}{|c|c|}
\hline Question I & $\begin{array}{l}\text { Do you know the difference between forgetfulness and } \\
\text { dementia? }\end{array}$ \\
\hline Question 2 & Do you think dementia is an illness? \\
\hline Question 3 & Do you know about the effects of donepezil? \\
\hline Question 4 & Do you know the side effects of donepezil? \\
\hline Question 5 & $\begin{array}{l}\text { Do you know that you must not stop donepezil even if } \\
\text { taking the drug does not cause any change in symptoms? }\end{array}$ \\
\hline Question 6 & $\begin{array}{l}\text { Do you know that you must not take two doses } \\
\text { together, even if you have forgotten to take a dose? }\end{array}$ \\
\hline
\end{tabular}

and whether or not it could delay progression of dementia and reduce the caregiver's burden. Question 4 was about possible side effects of donepezil, including gastrointestinal symptoms (such as vomiting and diarrhea) and mental symptoms (such as excitement and frustration). Question 5 was about the importance of continuing donepezil treatment even if there was no change in the symptoms of dementia. Question 6 asked how to handle a missed dose and the answer emphasized that it was incorrect to take two doses on one day.

The level of understanding was graded by giving a score of 1 for each "correct answer" and a score of 0 for each "wrong answer".

\section{Medication persistence rate}

Persistence with donepezil therapy was investigated for a maximum of 1 year from the start of treatment. Compliance was checked at each visit to the Hospital Pharmacy Department and the reasons for discontinuation of therapy (if any) were investigated.

The relationship between the medication persistence rate and CDR was investigated, as well as factors with a possible influence on persistence.

\section{Statistical analysis}

Pearson's Chi-square test was employed for analysis of categorical variables and the $t$-test was used for continuous variables.

Wilcoxon's signed-rank test was used to assess changes of understanding after consultation. The Cox proportional hazards model and Kaplan-Meier analysis were employed to estimate the medication persistence rate. The Cox proportional hazards model was performed to analyze factors that influenced medication persistence. Variables that showed a significant difference on univariate analysis were entered into a multivariate model. Statistical significance was accepted at $P<0.05$ in all analyses.

SPSS software (version 15.0; SPSS Inc, Chicago, IL) was used. 


\section{Ethical considerations}

The present study was conducted after receiving approval from the Ethical Review Boards of Nagoya University School of Medicine and Aichi Gakuin University School of Pharmacy. This study was conducted in accordance with the Declaration of Helsinki.

\section{Results}

The average duration of donepezil treatment was $248.6 \pm 184.1$ days in the non-DOCS group, while it was $379.0 \pm 202.6$ days in the DOCS group.

In the DOCS group, the mean duration of the first outpatient consultation was $46.4 \pm 7.2$ minutes and the mean duration of the second consultation (4 weeks later) was $27.8 \pm 6.1$ minutes.

\section{Level of understanding}

The level of understanding was surveyed in 52 patients and their caregivers before and 4 weeks after the first consultation. The score for understanding (mean \pm standard deviation) increased from $2.5 \pm 1.7$ before the DOCS consultation to $5.7 \pm 0.7$ after consultation $(P<0.001$, Wilcoxon's signed-rank test) (Figure 1A). Four weeks after the first DOCS consultation, the level of understanding was significantly higher for all questions $(P<0.001$, Wilcoxon's signed-rank test) (Figure 1B).

\section{Medication persistence rate and the reasons for discontinuation}

Among 59 patients in the non-DOCS group, 29 (49.2\%) continued to use donepezil for 1 year. The reasons for discontinuation in the other 30 patients included transfer to another hospital (11 patients), side effects ( 9 patients) (gastrointestinal symptoms such as abdominal pain, diarrhea, and nausea (5 patients); bradycardia (2 patients); headache (1 patient); and changes in the behavioral and psychological symptoms of dementia (1 patient)), unknown (9 patients), and lack of response (1 patient). Discontinuation due to gastrointestinal symptoms occurred at $21.0 \pm 11.1$ days after the start of treatment.

Among the 52 patients in the DOCS group, 38 patients (73.1\%) continued to take donepezil during the 1-year observation period. The reasons for discontinuation in the other 14 patients included transfer to another hospital ( 9 patients), side effects (3 patients) (gastrointestinal systems such as abdominal pain, diarrhea, and nausea [3 patients]; and headache and tremor [1 patient]), and unknown ( 2 patients). Discontinuation due to gastrointestinal symptoms occurred at $21.0 \pm 18.5$ days after the start of treatment.

We examined the association between persistence with donepezil treatment and age, sex, CDR, living alone, and attending the DOCS in patients who continued or discontinued treatment. As a result, there was a significantly higher medication persistence rate in the DOCS group $\left(P=0.01, \chi^{2}\right.$ test $)$ (Table 2$)$.

The Kaplan-Meier curve for continuation of donepezil therapy is shown in Figure 2. Investigation of the association between the medication persistence rate and attending the DOCS revealed a significantly higher persistence rate in patients who visited the DOCS ( $P=0.008$, log-rank test).

We also examined the influence of age, sex, CDR, living alone, and attendance at the DOCS on medication persistence by univariate analysis using the Cox proportional hazards
A

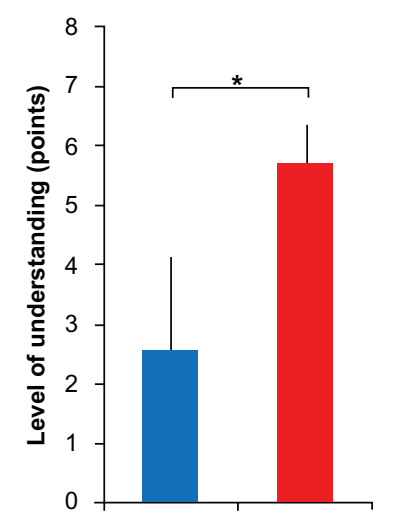

B

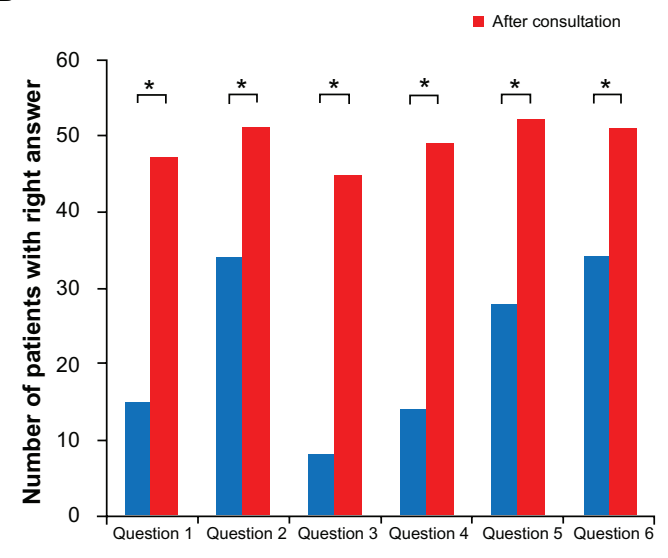

Figure I (A) Level of understanding about the clinical features of Alzheimer's disease and pharmacotherapy with donepezil in primary caregivers before and 4 weeks after the first DOCS consultation. (B) Number of primary caregivers giving correct answers about the clinical features of Alzheimer's disease and treatment with donepezil before and 4 weeks after the first DOCS consultation.

Notes: $* P<0.001$; Wilcoxon's signed-rank test.

Abbreviation: DOCS, Donepezil Outpatient Consultation Service. 
Table 2 Demographic characteristics and other factors compared between patients who continued and discontinued donepezil treatment

\begin{tabular}{llll}
\hline & $\begin{array}{l}\text { Patients who } \\
\text { continued } \\
\text { treatment }\end{array}$ & $\begin{array}{l}\text { Patients who } \\
\text { discontinued } \\
\text { treatment }\end{array}$ & P value \\
\hline $\begin{array}{l}\text { Age } \\
\text { Sex }\end{array}$ & $77.7 \pm 6.3$ & $78.9 \pm 6.5$ & 0.322 \\
$\quad$ Male & $19(52.8 \%)$ & $17(47.2 \%)$ & 0.258 \\
Female & $48(64.0 \%)$ & $27(36.0 \%)$ & \\
CDR & $11(68.8 \%)$ & $5(31.3 \%)$ & 0.357 \\
0.5 & $36(55.4 \%)$ & $29(44.6 \%)$ & \\
I & $11(57.9 \%)$ & $8(42.1 \%)$ & \\
2 & $9(81.8 \%)$ & $2(18.2 \%)$ & \\
3 & & $6(54.5 \%)$ & 0.287 \\
Living status & & \\
Living alone & $5(45.5 \%)$ & $38(38.0 \%)$ & \\
Living with others & $62(62.0 \%)$ & $30(50.8 \%)$ & 0.01 \\
Consultation & & $14(26.9 \%)$ & \\
Non-DOCS & $29(49.2 \%)$ & & \\
DOCS & $38(73.1 \%)$ & & \\
\hline
\end{tabular}

Abbreviations: CDR, clinical dementia rating; DOCS, Donepezil Outpatient Consultation Service.

model to assess the risk of discontinuation. The results indicated a significantly lower hazard ratio in the DOCS group, with the risk of discontinuation being 0.437 in the DOCS group relative to the non-DOCS group (Table 3).

\section{Discussion}

Donepezil was the first drug to be approved for the treatment of core symptoms of AD in Japan and it has been used as the mainstay of therapy for over a decade, but its effect is limited to retarding the progression of symptoms related to cognitive dysfunction. ${ }^{1,2}$ To fully benefit from this drug,

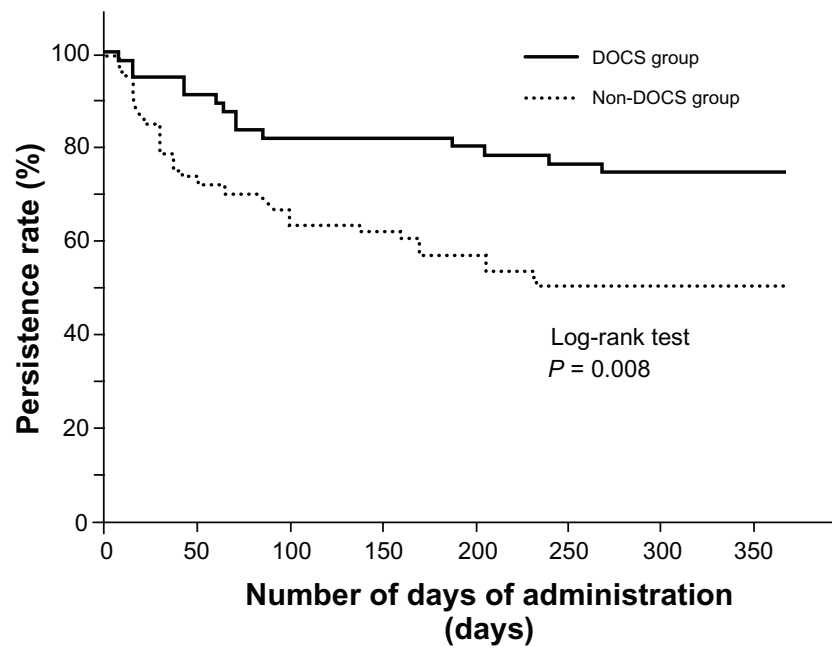

Figure 2 Persistence with treatment in the DOCS and non-DOCS groups. Abbreviation: DOCS, Donepezil Outpatient Consultation Service.
Table 3 Influence on medication persistence of age, gender, clinical dementia rating, living alone, and attending the DOCS assessed by Cox proportional hazards analysis

\begin{tabular}{lllll}
\hline & & Hazard ratio & $\mathbf{9 5 \%} \mathbf{C l}$ & P value \\
\hline Age & 1.022 & $0.973-1.072$ & 0.387 \\
Sex & 0.690 & $0.376-1.267$ & 0.232 \\
& 0.5 vs 3 & 2.100 & $0.407-10.828$ & 0.375 \\
CDR I vs 3 & 3.110 & $0.742-13.046$ & 0.121 \\
& 2 vs 3 & 3.096 & $0.657-14.589$ & 0.153 \\
\multicolumn{2}{l}{ Living alone } & 0.680 & $0.288-1.610$ & 0.381 \\
\multicolumn{2}{l}{ DOCS } & 0.437 & $0.231-0.824$ & 0.011 \\
\hline
\end{tabular}

Abbreviation: $\mathrm{CDR}$, clinical dementia rating; $\mathrm{Cl}$, confidence interval; $\mathrm{DOCS}$, Donepezil Outpatient Consultation Service.

it is important for patients to be diagnosed at an earlier stage and to promote adherence by the patients and their caregivers.

Umegaki et al reported that some $\mathrm{AD}$ patients discontinue donepezil treatment due to gastrointestinal side effects or lack of improvement of neurological symptoms, so that the therapeutic effect is inadequate. ${ }^{5}$

Physicians and pharmacists do not always have sufficient time to provide detailed information about medications, making it difficult for patients and their caregivers to fully understand the properties of drugs. Accordingly, a consultation service provided by pharmacists, such as the DOCS, can help patients and their caregivers to understand the clinical features of $\mathrm{AD}$ and treatment with donepezil.

We surveyed the primary caregivers of the AD patients prior to consultation at the DOCS to assess their knowledge about the disease and donepezil therapy. As a result, most of them gave incorrect answers to questions 3 and 4 (concerning the effects and side effects of donepezil), and to question 5 (Do you know that you must not stop donepezil even if the drug does not cause any change in symptoms?).

These findings corresponded well with the reasons for discontinuation identified in our previous study. Before attending the DOCS, caregivers may have had excessively high expectations regarding the effects of treatment, so that misgivings about the lack of improvement of dementia symptoms or alleviation of the caregiver burden, as well as concern about side effects, are likely to have influenced adherence.

Understanding of $\mathrm{AD}$ was also surveyed at four weeks after initial visit to the DOCS by asking caregivers the same questions again. As a result, their understanding of all issues showed significant improvement.

Kuzuya et al reported that compliance by elderly people is associated with their understanding of a treatment 
regimen and drug actions, with instruction about dosage and administration being important. ${ }^{8}$ In the same way, improved understanding of donepezil therapy increased the medication persistence rate in the present study.

With regard to the gastrointestinal side effects of donepezil treatment, an early onset was noted (at 21.0 \pm 11.1 days in the non-DOCS group and 21.0 \pm 18.5 in the DOCS group). Gastrointestinal side effects (nausea, anorexia, and diarrhea) of donepezil have been reported to occur after treatment at $3 \mathrm{mg} /$ day (its starting dose) or after dose escalation to $5 \mathrm{mg} /$ day (its effective dose), ${ }^{9-11}$ consistent with the pattern of occurrence in the present study.

We scheduled the second DOCS consultation at four weeks after the initial session, but this is after the period when gastrointestinal side effects are likely to develop, so adequate management of these symptoms may not be achieved.

Also, an explanation about specific treatments for gastrointestinal side effects of donepezil was not given at the DOCS. Accordingly, information on treating these side effects should be prepared and a system should be organized to allow rapid contact with a pharmacist if side effects occur.

In this way, pharmacists can provide treatment support that is tailored according to the individual condition and lifestyle of a patient, which is important to improve adherence.

It has been reported that the medication persistence rate for acetylcholinesterase inhibitors is lower in AD patients with no social support. ${ }^{12}$ Therefore, we provided information on various services and supports, including healthcare activities, medical services, and welfare, to the patients and their caregivers in cooperation with medical social workers when they visited the DOCS.

In this study, the medication persistence rate was significantly higher in the DOCS group compared with the nonDOCS group (73.1\% vs 49.2\%), and donepezil treatment was continued in the DOCS group regardless of other factors that could be expected to influence medication persistence (age, sex, CDR, and living alone).

The medication persistence rate is generally reported to be about $30 \%-60 \%$ when acetylcholinesterase inhibitors are used to treat $\mathrm{AD},{ }^{11-15}$ and medication persistence has been reported to decrease significantly as the CDR increases in patients who have been prescribed donepezil. ${ }^{5}$ However, our findings suggest that advice on dosage and administration provided by pharmacists at a service like the DOCS can improve adherence.

A limitation of this study was that we investigated outpatients of university hospitals. Most patients who attend university hospitals have been referred from other hospitals and may have more advanced dementia compared with patients at general hospitals. Therefore, our results may not be replicated by studies conducted at general hospitals.

Another issue is that 20 out of 111 patients who discontinued donepezil therapy did so because of transfer to another hospital/clinic (non-DOCS group: 11, DOCS group: 9). Medication persistence was not followed up in these patients after transfer, which may have affected the accuracy of our results. Nevertheless, we consider that the present study demonstrates the value of involving pharmacists in dementia care and confirms the positive effect of the DOCS, which could be a model applicable to local dispensing pharmacies for providing support to patients' families and improving medication persistence. Close collaboration between the hospital pharmacy department and local dispensing pharmacies is the key to establishing such a system that could provide a substantial contribution to dementia care.

In January 2011, memantine and galantamine were granted approval for treatment of the core symptoms of AD in Japan. Accordingly, treatment of AD is expected to become more diverse, making the involvement of pharmacists even more important in the future.

\section{Acknowledgments}

We gratefully acknowledge the assistance of Dr Keiko Yamamura (Clinical pharmaceutical course, Aichi Gakuin University), Dr Kiyofumi Yamada (Professor, Pharmacy Department, Nagoya University Hospital), and Dr Yusuke Suzuki (Lecturer, Geriatric Internal Medicine Division, Nagoya University School of Medicine).

\section{Disclosure}

The authors report no conflicts of interest in this work.

\section{References}

1. Homma A, Takeda M, Imai Y, et al. Clinical efficacy and safety of donepezil on cognitive and global function in patients with Alzheimer's disease. A 24-week, multicenter, double-blind, placebo-controlled study in Japan. E2020 Study Group. Dement Geriatr Cogn Disord. 2000;11(6): 299-313.

2. Rogers SL, Doody RS, Pratt RD, Ieni JR. Long-term efficacy and safety of donepezil in the treatment of Alzheimer's disease: final analysis of a US multicentre open-label study. Eur Neuropsychopharmacol. 2000; 10(3):195-203.

3. Mohs RC, Doody RS, Morris JC, et al. A 1-year, placebo-controlled preservation of function survival study of donepezil in $\mathrm{AD}$ patients. Neurology. 2001;57(3):481-488.

4. Wimo A, Winblad B, Shah SN, Chin W, Zhang R, McRae T. Impact of donepezil treatment for Alzheimer's disease on caregiver time. Curr Med Res Opin. 2004;20(8):1221-1225.

5. Umegaki H, Itoh A, Suzuki Y, Nabeshima T. Discontinuation of donepezil for the treatment of Alzheimer's disease in geriatric practice. Int Psychogeriatr. 2008;20(4):800-806. 
6. American Psychiatric Association. Diagnostic and Statistical Manual of Mental Disorder. 4th ed. Washington: American Psychiatric Association; 1994.

7. Kuzuya M, Enoki H, Izawa S, et al. Prevalence of oral intake difficulty and associated factors in disabled elderly. The Journal of Japanese Society for Parenteral and Enteral Nutrition. 2011;26: 1265-1270.

8. Kuzuya M, Endo H, Umegaki H, et al. Factors influencing noncompliance with medication regimens in the elderly. Nihon Ronen Igakkai Zasshi. 2000;37(5):363-370.

9. Homma A, Imai Y, Hariguchi S, et al. Late Phase II Clinical Study of Acetylcholinesterase Inhibitor E2020 in Patients with Alzheimertype Dementia. 12-weeks Double-blind, Placebo-Controlled Study, $3 \mathrm{mg} /$ day, $5 \mathrm{mg}$ /day. Clinical Evaluation. 1998;26:251-284.

10. Inglis F. The tolerability and safety of cholinesterase inhibitors in the treatment of dementia. Int J Clin Pract Suppl. 2002;127:45-63.
11. Kröger E, van Marum R, Souverein P, Egberts T. Discontinuation of cholinesterase inhibitor treatment and determinants thereof in The Netherlands: A retrospective cohort study. Drugs Aging. 2010;27(8):663-675.

12. Amuah JE, Hogan DB, Eliasziw M, et al. Persistence with cholinesterase inhibitor therapy in a population-based cohort of patients with Alzheimer's disease. Pharmacoepidemiol Drug Saf. 2010;19(7):670-679.

13. Frankfort SV, Appels BA, de Boer A, et al. Discontinuation of rivastigmine in routine clinical practice. Int J Geriatr Psychiatry. 2005;20(12): 1167-1171.

14. Abughosh SM, Kogut SJ. Comparison of persistence rates of acetylcholine-esterase inhibitors in a state Medicaid program. Patient Prefer Adherence. 2008;2:79-85.

15. Herrmann N, Binder C, Dalziel W, Smyth S, Camacho F. Persistence with cholinesterase inhibitor therapy for dementia: an observational administrative health database study. Drugs Aging. 2009;26(5): 403-407.

\section{Publish your work in this journal}

Patient Preference and Adherence is an international, peer-reviewed, open access journal focusing on the growing importance of patient preference and adherence throughout the therapeutic continuum. Patient satisfaction, acceptability, quality of life, compliance, persistence and their role in developing new therapeutic modalities and compounds to optimize clinical outcomes for existing disease states are major areas of interest. This journal has been accepted for indexing on PubMed Central. The manuscript management system is completely online and includes a very quick and fair peer-review system. Visit http://www.dovepress.com/ testimonials.php to read real quotes from published authors.

Submit your manuscript here: http://www.dovepress.com/patient-preference-and-adherence-journal 